\title{
Refractory Myasthenia Gravis - Patient Burden and the Need for New Therapeutic Targets
}

\author{
Saiju Jacob \\ Queen Elizabeth Neuroscience Centre, University Hospitals of Birmingham NHS Foundation Trust, Birmingham, UK
}

$\mathrm{M}$ yasthenia gravis (MG) is an autoimmune disorder characterised by muscle weakness and fatigue. Symptoms include slurred speech, weakness in the arms and legs and difficulty swallowing and breathing, which can lead to life-threatening myasthenic crisis. Despite receiving recommended therapies, many patients with MG report health-related quality of life is low, largely due to impaired mobility and depression. Furthermore, around 10\% of patients with generalised MG fail to respond to treatment. Patients with refractory MG have a severely compromised quality of life and it is important that new treatments are evaluated for this group of patients.

\section{Keywords}

Refractory myasthenia gravis, quality of life

Disclosure: Saiju Jacob reports receiving personal compensation from Alexion Pharmaceuticals serving as an international advisory board member: Saiju Jacob is also a clinical tria investigator for Alexion Pharmaceuticals.

Review Process: This article is a short opinion piece and has not been submitted to external peer reviewers. It was reviewed by a member of the editorial board before publication. The article was also reviewed for scientific accuracy by Alexion Pharmaceuticals.

Acknowledgements: Medical writing assistance, including preparation of the drafts under the guidance of the author, was provided by Katrina Mountfort for Touch Medical Media and was supported by Alexion Pharmaceuticals.

Authorship: The named author meets the Internationa Committee of Medical Journal Editors (ICMJE) criteria for authorship of this manuscript, takes responsibility for the integrity of the work as a whole, and has given final approval for the version to be published.

Open Access: This article is published under the Creative Commons Attribution Noncommercial License, which permits any non-commercial use, distribution, adaptation and reproduction provided the original author and source are given appropriate credit. () The Author 2018.

Received: 19 January 2018

Published Online: 6 June 2018

Citation: European Neurological

Review. 2018:13(1):18-20

Corresponding Author: Saiju Jacob, Consultant Neurologist and Clinical Lead, Queen Elizabeth Neuroscience Centre, University Hospitals of Birmingham NHS Foundation Trust, Birmingham, B15 2TH, UK. E: Saiju.Jacob@uhb.nhs.uk

Support: The publication of this article was supported by Alexion Pharmaceuticals. The author received no funding or compensation for development and authorship of this article. The views and opinions expressed in the article are those of the author and not necessarily those of Alexion Pharmaceuticals.
Myasthenia gravis (MG) is a rare autoimmune disease that affects around 11-32 people per $100,000 .^{1-3}$ Because of improved recognition and diagnosis, its incidence is increasing. ${ }^{4.5}$ The disease can occur at any age but its onset is most common in women before the age of 40 and men after the age of $60 .{ }^{6}$ Although the number of patients affected is relatively small, the impact of MG on patients, their families, and society is profound. Typically, symptoms involve muscle weakness that worsens after periods of activity and improves after periods of rest.

Myasthenia gravis typically presents with a specific muscular weakness rather than generalised weakness.' It often involves only the ocular muscles and presents as intermittent ptosis (eyelid droop) and diplopia (double vision). ${ }^{6-8}$ In around two-thirds of patients, the disease progresses over weeks to months with exacerbations and remissions, leading to bulbar symptoms such as dysphagia, dysphonia, dysarthria, chewing difficulty and weakness of the limbs and axial muscles (Figure 1). ${ }^{8}$ Around $87 \%$ of patients progress to generalised disease within 13 months of initial onset ${ }^{7}$ and, in the majority of patients, symptom onset to maximal weakness occurs within the first 2 years. ${ }^{9}$

Despite a large number of therapeutic options, around $10 \%$ of patients with MG suffer from treatment-refractory disease..$^{10}$ Definitions of refractory MG vary, but patients with refractory MG typically experience frequent clinical relapses upon lowering their immunotherapy, are clinically unstable on their current immunotherapeutic treatment regimen or develop severe side effects from immunotherapy? ${ }^{710}$ Several studies have investigated the characteristics associated with refractory disease. A retrospective single centre study $(n=128)$ identified 19 patients with treatment-refractory MG (14.8\%), defined as those who could not lower their immunotherapy without clinical relapse, were not clinically controlled on their immunotherapy regimen, or experienced severe side effects from immunotherapy."1 These were more likely to be younger at onset (Figure 2), female, associated with thymoma, and muscle-specific kinase (MuSK) antibody positive. In addition, a significantly higher proportion of patients with refractory MG had undergone thymectomy ( $68 \%$ versus $17 \%, p<0.001$ ), suggesting underlying biological differences between patients with refractory and non-refractory MG. ${ }^{11,12}$ These findings were confirmed in another retrospective study involving seven centres $(n=512)$, in which 76 patients $(14.8 \%)$ were considered to be refractory. Characteristics associated with patients with refractory MG were similar and included MuSK antibody positivity (relative risk [RR] 9.57; $p<0.0001$ ), female gender (RR 2.69, $p<0.0001$ ), having undergone thymectomy (RR 3.95; $p<0.0001$ ), presence of thymoma (RR 2.91, $p=0.0001$ ), and younger age at onset (median age of patients with refractory MG was 36 versus 61 years in patients with non-refractory MG). Patients with refractory MG also had a higher prevalence and poor control of type 2 diabetes ( $\mathrm{HbA} 1 \mathrm{C}>8 \%$ ) and dyslipidaemia, which may be due to increased steroid usage, adding to the disease burden.? 
Figure 1. Health burden of myasthenia gravis

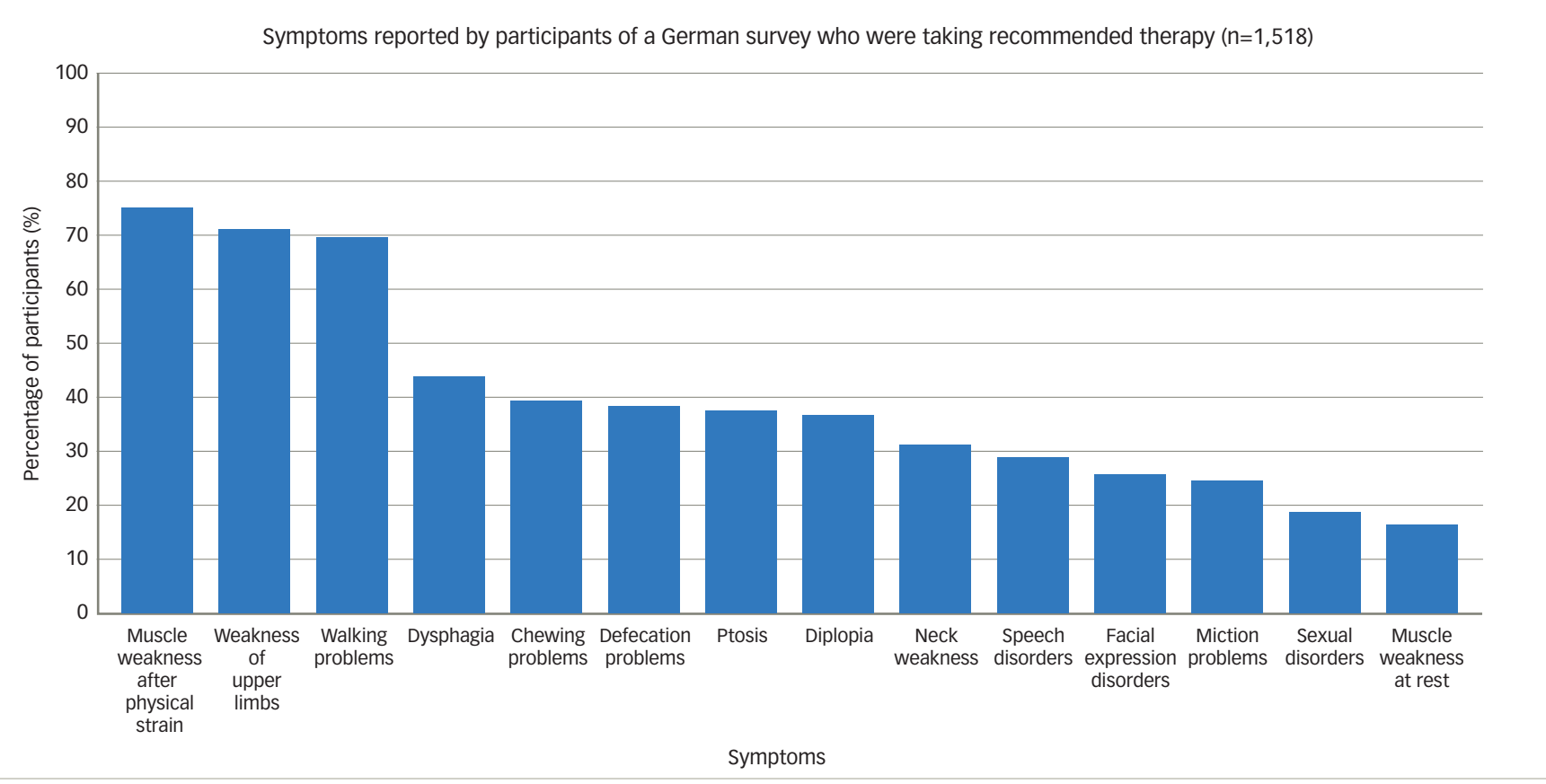

Data source: Twork et al., 2010.12

The current international consensus guidance, according to the Myasthenia Gravis Foundation of America, defines refractory disease as follows:

'Post-intervention status is unchanged or worse after corticosteroids and at least 2 other immunosuppressive agents, used in adequate doses for an adequate duration, with persistent symptoms or side effects that limit functioning, as defined by patient and physician. ${ }^{\prime 13}$

However, this is not a true consensus statement, as many guidelines in different countries do not share these recommendations or do not give a definition of refractory disease. ${ }^{14,15}$ Patients may remain refractory for more than 10 years: ${ }^{16}$ they are often considered refractory until they respond to a therapy or become asymptomatic without therapeutic intervention.

Patients with refractory MG periodically experience acute exacerbations of their symptoms, including life-threatening swallowing impairment. When the muscles necessary for breathing are affected, a patient is said to be in myasthenic crisis. This affects $15-20 \%$ of patients with MG and can result in respiratory failure, necessitating intubation and mechanical ventilation. ${ }^{17}$ In an Australian survey ( $\left.n=190\right), 19 \%$ of participants had required the support of a ventilator due to $M \mathrm{MG}^{18}$

As well as the immediate medical risk, patients with refractory MG have a severely compromised quality of life. ${ }^{12,18-21}$ Patients with MG also experience difficulty performing everyday activities due to weakness in the muscles, especially limbs. Around a third of patients cannot drive, either because of visual impairments or inability to hold the steering wheel due to weak arms. ${ }^{18}$ Patients have difficulty with tasks such as climbing stairs, hanging the washing and everyday housework. ${ }^{18} \mathrm{~A}$ third of patients with MG require their spouse or partner to be their carer as they are no longer able to care for themselves, imposing financial burdens. ${ }^{18}$ Patients often suffer from diplopia, which interferes with most activities of daily living. 12,18 Weakness of the facial and bulbar muscles may result in inability to speak, chew and swallow, or difficulty holding up their head because of neck muscle weakness. ${ }^{12,18}$
Figure 2. Age distribution of patients with refractory versus non-refractory myasthenia gravis

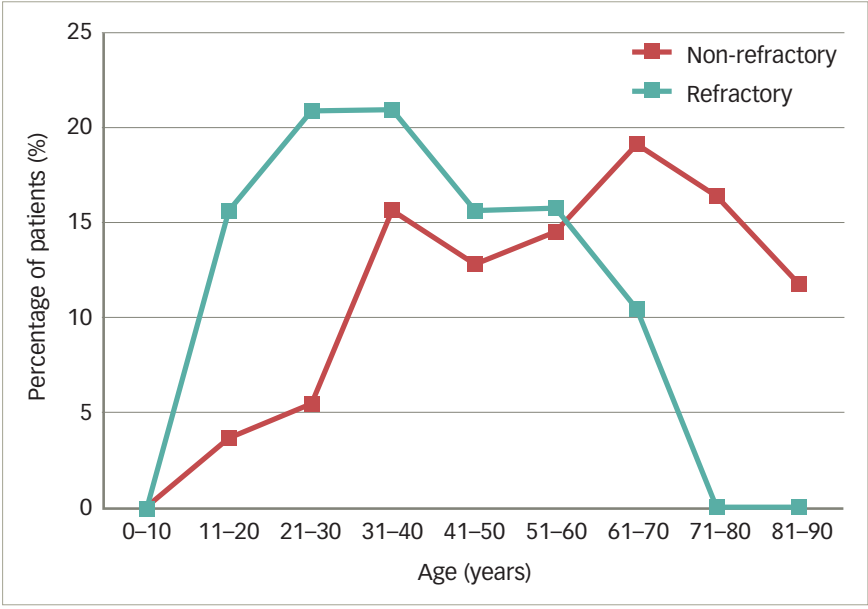

Data source: Suh et al., 2013. ${ }^{11}$

The emotional burden of refractory MG is also considerable. Patients may have to give up their careers and report feelings of isolation. . $8,22^{2}$ Depression has been reported in around one third of patients with $M G^{12,18}$ and refractory disease has been identified as a factor associated with depression in MG. ${ }^{23}$ A Danish study $(n=330)$ found that patients with MG had almost six times higher odds of unemployment and almost nine times higher odds of long-term sick leave 2 years after diagnosis, compared with the general population..$^{24} \mathrm{~A}$ recent Japanese study $(\mathrm{n}=917)$ reported that $27 \%$ of patients with MG had experienced unemployment, 4.1\% had unwillingly accepted job transfers and $35.9 \%$ had a decrease in income, $47.1 \%$ of whom reported that the decrease was more than half of their previous total income. In addition, almost half of the patients reported feeling reduced social positivity. ${ }^{25}$

To reduce the social disadvantages associated with MG and its treatment, a greater focus is needed on helping patients to achieve treatment goals, 
particularly patients with refractory MG. Commonly used therapies have had a limited impact on refractory disease and up to $15 \%$ of patients either fail to respond to treatment or suffer intolerable side effects. For such patients, there is a need for more aggressive treatment or treatment directed to the underlying pathogenesis of the disease in order to prevent life-threatening crises, restore muscular strength and improve quality of life.
In conclusion, refractory MG is rare but has a severely detrimental effect of the lives of patients and their carers. While refractory MG is not a major public health problem in terms of number of patients affected, it is a chronic problem with a major financial impact on the patients themselves and the care system. There is a need not only for more evidence-based therapies but also on other measures that might enhance quality of life in patients with MG.
1. Breiner A, Widdifield J, Katzberg HD, et al. Epidemiology of myasthenia gravis in Ontario, Canada. Neuromuscul Disord. 2016;26:41-6

2. Santos $E$, Coutinho $E$, Moreira I, et al. Epidemiology of myasthenia gravis in Northern Portugal: Frequency estimates and clinical epidemiological distribution of cases. Muscle Nerve. 2016;54:413-21.

3. Phillips $L \mathrm{LH}, 2$ nd. The epidemiology of myasthenia gravis. Ann $\mathrm{N}$ Y Acad Sci. 2003;998:407-12.

4. Phillips LH, 2nd, Torner JC. Epidemiologic evidence for a changing natural history of myasthenia gravis. Neurology. 1996;47:1233-8

5. Carr AS, Cardwell CR, McCarron PO, McConville J. A systematic review of population based epidemiological studies in myasthenia gravis. BMC Neurol. 2010;10:46.

6. National Institutes of Health. Myasthenia Gravis fact sheet 2017. Available at: https://www.ninds.nih.gov/Disorders/ Patient-Caregiver-Education/Fact-Sheets/Myasthenia-GravisFact-Sheet (accessed 8 August 2017).

7. Sudulagunta SR, Sepehrar M, Sodalagunta MB, et al. Refractory myasthenia gravis - clinical profile, comorbidities and response to rituximab. Ger Med Sci. 2016;14:Doc12.

8. Silvestri NJ, Wolfe GI. Myasthenia gravis. Semin Neurol. 2012;32:215-26.

9. Grob D, Brunner N, Namba T, Pagala M. Lifetime course of myasthenia gravis. Muscle Nerve. 2008:37:141-9.
10. Silvestri NJ, Wolfe GI. Treatment-refractory myasthenia gravis. J Clin Neuromuscul Dis. 2014;15:167-78

11. Suh J, Goldstein JM, Nowak RJ. Clinical characteristics of refractory myasthenia gravis patients. Yale J Biol Med. 2013;86:255-60.

12. Twork $\mathrm{S}$, Wiesmeth $\mathrm{S}$, Klewer J, et al. Quality of life and life circumstances in German myasthenia gravis patients. Health Qual Life Outcomes. 2010;8:129.

13. Sanders DB, Wolfe $\mathrm{Gl}$, Benatar $\mathrm{M}$, et al. International consensus guidance for management of myasthenia gravis: Executive summary. Neurology. 2016;87:419-25.

14. Murai H. Japanese clinical guidelines for myasthenia gravis: Putting into practice. Clin Exp Neuroimmunol. 2015;6:21-31.

15. Sussman J, Farrugia ME, Maddison P, et al. Myasthenia gravis: Association of British Neurologists' management guidelines. Pract Neurol. 2015;15:199-206.

16. Matsuo K, Fujii, C, Miyata, K, et al. A case of refractory generalized myasthenia gravis treated with polypharmacy and plasma exchange. J Neurol Neurophysiol. 2016;7:366.

17. Wendell $L C$, Levine JM. Myasthenic crisis. Neurohospitalist. 2011;1:16-22.

18. The Centre for International Economics. Final report: the cost to patients and the community of Myasthenia Gravis. 2014. Available at: http://www.thecie.com.au/wp-content/ uploads/2014/06/Final-report_Economic-Impact-ofMyasthenia-Gravis-08112013.pdf (accessed 9 August 2017).
19. Boldingh MI, Dekker L, Maniaol AH, et al. An up-date on health-related quality of life in myasthenia gravis -results from population based cohorts. Health Qual Life Outcomes. 2015;13:115.

20. Yang Y, Zhang $\mathrm{M}$, Guo J, et al. Quality of life in 188 patients with myasthenia gravis in China. Int J Neurosci. 2016;126:455-62.

21. Basta IZ, Pekmezović TD, Perić SZ, et al. Assessment of healthrelated quality of life in patients with myasthenia gravis in Belgrade (Serbia). Neurol Sci. 2012;33:1375-81.

22. Howard JF, Jr. Rare insight: a physician's perspective on myasthenia gravis. 2016. Available at: https://globalgenes.org/ raredrily gre insight . Anysicians-perspective-on-myastheni gravis/ (accessed 8 August 2017)

23. Suzuki Y, Utsugisawa K Suzuki S, et al. Factors associated with depressive state in patients with myasthenia gravis: a multicentre cross-sectional study. BMJ Open. 2011;1:e000313. multicentre cross-sectional study. BM
DOI: 10.1136/bmjopen-2011-000313

24. Frost A, Svendsen ML, Rahbek J, et al. Labour market participation and sick leave among patients diagnosed with myasthenia gravis in Denmark 1997-2011: a Danish nationwide cohort study. BMC Neurol. 2016;16:224

25. Nagane $Y$, Murai $H$, Imai $T$, et al. Social disadvantages associated with myasthenia gravis and its treatment: a multicentre crosssectional study. BMJ Open. 2017;7:e013278. DOI: 10.1136/ bmjopen-2016-013278 